\title{
Flujo Óptimo Reactivo mediante Optimización por Enjambre de Partículas
}

Daniel Gutiérrez, Walter M. Villa y Jesús M. López-Lezama*

Grupo de Investigación GIMEL, Departamento de Ingeniería Eléctrica, Facultad de Ingeniería, Universidad de Antioquia, Calle 67 No. 53-108, Medellín - Colombia (e-mail:

daniel.gutierrezr@udea.edu.co; walter.villa@udea.edu.co; jmaria.lopez@udea.edu.co;).

${ }^{*}$ Autor a quien debe ser dirigida la correspondencia.

Recibido Feb. 13, 2017; Aceptado Abr. 6, 2017; Versión final May. 9, 2017, Publicado Oct. 2017

\section{Resumen}

En este artículo se explora la optimización por enjambre de partículas (PSO) con algunas de sus variantes para dar solución al problema de flujo de potencia óptimo reactivo ORPD. Este problema consiste consiste en usar de forma óptima los recursos de potencia reactiva de un sistema de potencia, comúnmente con el objetivo de minimizar las pérdidas de energía y mejorar el perfil de tensiones. El ORPD es un problema no lineal y no convexo que involucra variables continuas y enteras y que presenta múltiples soluciones subóptimas. Además de la técnica PSO las variantes conocidas como Turbulent PSO (TPSO) y Turbulent Crazy PSO (TCPSO) fueron implementadas. Se realizaron varias pruebas en los sistemas IEEE de 57 y 118 barras comparando los resultados con otros reportados en la literatura técnica. Se encontró que el desempeño del PSO se mejora considerablemente cuando se aplican las variantes TPSO y TCPSO.

Palabras clave: flujo óptimo reactivo; optimización por enjambre de partículas; sistemas de potencia

\section{Optimal Reactive Power Dispatch by means of Particle Swarm Optimization}

\begin{abstract}
In this paper the particle swarm optimization (PSO) along with some of its variants is explored to give solution to the optimal reactive power dispatch problem (ORPD). This problem consists of the optimal use of reactive power resources in a power system, usually with the aim of minimizing power loses and improving voltage profile. The ORPD is a non-lineal and non-convex problem that involves continues and discrete variables and features multiple sub-optimal solutions. Besides the PSO technique, the variants known as Turbulent PSO (TPSO) and Turbulent Crazy (PSO) were implemented. Several tests were performed on IEEE 57 and 118 bus test systems comparing results with other reported in the technical literature. It was found that the performance of PSO is considerably improved when the variants TPSO and TCPSO are applied.
\end{abstract}

Keywords: optimal reactive power flow; particle swarm optimization; power systems 


\section{INTRODUCCIÓN}

El despacho de fuentes de potencia reactiva y ajuste óptimo de tensiones en las barras de generación es lo que se conoce como flujo de potencia óptimo reactivo (ORPD por sus siglas en inglés). El ORPD es un problema complementario al flujo óptimo de potencia activa, que se lleva a cabo después de este y cuyo objetivo principal es la minimización de pérdidas y mejora del perfil de tensiones. Las variables de control del ORPD son las posiciones de las tomas de derivación de transformadores, inyecciones de potencia reactiva de bancos de capacitores y ajustes en las magnitudes de tensión en las barras de generación. En la Fig. 1 se presenta una ilustración del ORPD. Se parte de un sistema de potencia en un punto de operación dado y se llega a un punto de operación diferente con pérdidas menores a las iniciales mediante la gestión de reactivos. Las primeras tentativas para solucionar el ORPD se basaron en técnicas de programación matemática clásica. Si bien estas técnicas son rápidas desde el punto de vista computacional, no tienen un buen desempeño cuando abordan problemas de naturaleza no convexa que involucran variables discretas. Adicionalmente, presentan dificultades para manejar problemas de gran dimensión. Por lo anterior, en la actualidad el ORPD se aborda principalmente mediante técnicas de programación metaheurísticas. Estas técnicas pueden abordar problemas de naturaleza no convexa y multimodal (con posibilidad de múltiples óptimos locales). Si bien estas técnicas no garantizan la obtención de un óptimo global permiten encontrar soluciones de alta calidad en tiempos computacionales aceptables (Granada, et al., 2009).

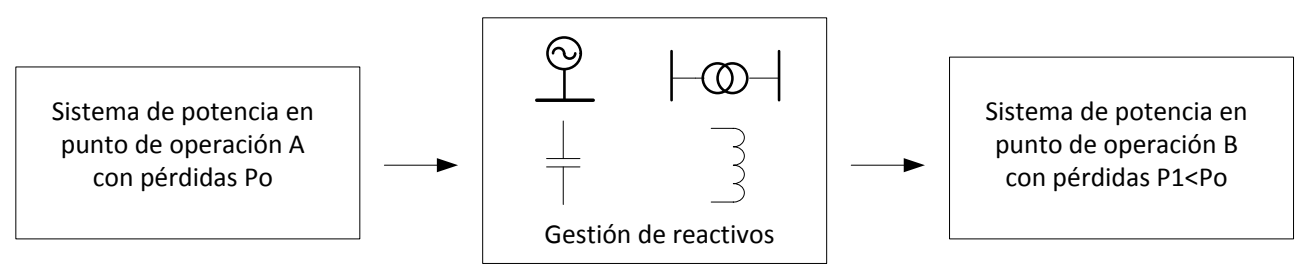

Fig.1: Representación del ORPD.

En la literatura técnica se han propuesto diferentes metaheurísticas para solucionar el ORPD. En (Vlachogiannis y Lee, 2008), (Varadarajan y Swarup, 2008) y (Xuexia et al., 2012) se presentan algoritmos evolutivos para abordar el ORPD. Estos algoritmos se basan en los postulados de la evolución biológica, en la que un conjunto inicial de individuos (candidatos de solución) dan origen a otros individuos con los que deben competir de tal forma que los más aptos (soluciones de mejor calidad) prevalezcan a lo largo del tiempo, dando origen a nuevas y mejores soluciones. En (Vlachogiannis y Lee, 2008) se presenta un algoritmo evolutivo basado en computación cuántica que busca resolver en forma conjunta el despacho óptimo de potencia activa y reactiva. Por otro lado, en (Varadarajan y Swarup, 2008) se propone un algoritmo diferencial (DE), para abordar el ORPD mientras en (Xuexia et al., 2012) se aborda el ORPD desde el punto de vista multi-objetivo para mejorar tensiones y reducir pérdidas de potencia.

En (Binod et al, 2014), (Liang y Wang, 2003) y (Khazali y Kalantar, 2011) se utilizan algoritmos basados en fenómenos físicos para resolver el ORPD. Estos algoritmos se inspiran en leyes de la física como la gravedad, las cargas eléctricas, el recocido del acero y cerámicas, etc. En (Binod et al., 2014) se presenta un algoritmo de búsqueda gravitacional con el objetivo de minimizar las pérdidas de potencia. En esta técnica los agentes (candidatos de solución) son representados mediante masas que interactúan según la ley de la gravedad y las leyes de Newton. En (Liang y Wang, 2003) se presenta un algoritmo basado en recocido simulado para resolver el ORPD con el objetivo de mejorar los niveles de tensión de una red de distribución. En (Khazali y Kalantar, 2011) se aborda el ORPD mediante una búsqueda armónica con el objetivo de minimizar pérdidas de potencia y mejorar perfiles de tensión. Este método se basa en un fenómeno inspirado en el proceso de improvisación de los músicos.

En (Hong et al., 2014) y (Martínez-Rojas et al., 2011) se utiliza la optimización por enjambre de partículas (PSO por sus siglas en inglés) para resolver el ORPD. Este método permite resolver un problema de optimización combinatoria modelando las posibles soluciones como partículas dentro de un enjambre. Dichas partículas se mueven dentro del espacio de búsqueda obedeciendo un conjunto de reglas que tienen en cuenta su posición y velocidad. La combinación de varias técnicas también es común en la solución del ORPD. En (Kanna y Singh, 2015) y (Srivastava y Singh, 2015) se presentan métodos híbridos para solucionar el ORPD. En (Vlachogiannis, y Kwang, 2006) y (Ghasemi et al., 2015) se presentan estudios comparativos de técnicas de solución utilizadas en el ORPD. Una revisión detallada del estado del arte sobre metaheurísticas aplicadas al ORPD puede ser consultada en (Gutiérrez, et al., 2016).

Si bien el ORPD se ha abordado usando PSO, algunas de sus variantes no han sido exploradas; lo cual constituye la principal contribución de este artículo. En este sentido, el objetivo de este trabajo es contribuir a la discusión sobre la aplicación de las técnicas de optimización metaheurística al ORPD. Para probar la 
efectividad de la técnica propuesta se utilizaron los sistemas de prueba IEEE de 57 y 118 barras. Los resultados evidencian la aplicabilidad y efectividad de la técnica propuesta y sus variantes, pues se obtienen soluciones de alta calidad; y en algunos casos superiores a las obtenidas con otros métodos reportados en la literatura técnica.

\section{MODELO MATEMÁTICO}

El modelo matemático del ORPD se presenta en tres secciones, para mayor claridad y comprensión: en la primera se plantea la función objetivo, en la segunda se aclaran las restricciones de igualdad, y en la tercera se describen las restricciones de desigualdad.

\section{Función Objetivo}

La función objetivo consiste en la minimización de pérdidas dada por la ecuación (1). En este caso Ploss representa las pérdidas de potencia, $g_{k}$ es la conductancia de la línea $k, V_{i}$ y $V_{j}$ son las magnitudes de tensión en las barras $i$ y $j$, respectivamente, $\theta_{i j}$ es la apertura angular de las barras $i$ y $j$; finalmente, $N_{K}$ es el conjunto de ramas del sistema. El ORPD es un problema de optimización de la operación del sistema de potencia. Por lo tanto, no se tienen en cuenta los costos de inversión de los equipos que proporcionan potencia reactiva. La ubicación y dimensionamiento de dichos elementos pertenece a otro problema de optimización (planeamiento de la expansión) que debe llevarse a cabo con anterioridad al ORPD.

$$
\operatorname{Min} P_{\text {loss }}=\sum_{k \in N_{K}} g_{k}\left(V_{i}^{2}+V_{j}^{2}-2 V_{i} V_{j} \cos \theta_{i j}\right)
$$

\section{Restricciones de igualdad}

Las restricciones de igualdad del ORPD están dadas por las ecuaciones nodales de balance de potencia activa y reactiva descritas por las ecuaciones (2) y (3), respectivamente. En este caso $\mathrm{N}_{B}$ representa el conjunto de barras del sistema; $G_{i j}$ y $B_{i j}$ son los valores real e imaginario de la posición $i, j$ de la matriz de admitancia nodal, respectivamente; $P_{g i}$ y $Q_{g i}$ son las potencias activa y reactiva generadas en la barra $i$, mientras que $P_{d i}$ y $Q_{d i}$ representan las potencias activa y reactiva demandadas en la misma barra, respectivamente. $Q_{c i}$ y $Q_{r i}$ representan la potencia reactiva inyectada por bancos de capacitores y reactores, respectivamente. Esta potencia se da en pasos discretos desde el mínimo hasta el máximo permitido para cada tipo de elemento.

$$
\begin{aligned}
& V_{i} \sum_{j \in N_{B}} V_{j}\left[G_{i j} \cos \theta_{i j}+B_{i j} \sin \theta_{i j}\right]-P_{g i}+P_{d i}=0 \\
& V_{i} \sum_{j \in N_{B}} V_{j}\left[G_{i j} \sin \theta_{i j}+B_{i j} \cos \theta_{i j}\right]-Q_{g i}-Q_{c i}+Q_{r i}+Q_{d i}=0
\end{aligned}
$$

\section{Restricciones de desigualdad}

Las restricciones de desigualdad del ORPD están dadas por las ecuaciones (4) a (10). Donde $N_{T}, N_{c} N_{R}$ y $N_{G}$ representan el número de transformadores con cambio de tomas, bancos de condensadores, bancos de reactores y el número de generadores del sistema, respectivamente. Por otro lado, los superíndices min y max hacen referencia a los límites mínimos y máximos de sus correspondientes variables. Las ecuaciones (4) y (5) representan los límites de tensión y potencia reactiva de los generadores, respectivamente. $V_{g i}$ y $Q_{g i}$ son la magnitud de tensión y generación de potencia reactiva del generador $i$, respectivamente. La ecuación (6) representa los límites del movimiento de tomas de derivación de los transformadores donde $T_{i}$ representa la posición del toma de derivación del transformador $i$. Las ecuaciones (7) y (8) representan los límites de inyección de potencia reactiva de los bancos de capacitores y reactores, respectivamente. En este caso $Q_{c i}$ y $Q_{r i}$ representan la potencia reactiva inyectada por el elemento capacitor e inductor $i$, respectivamente. Finalmente, las restricciones (9) y (10) son conocidas como restricciones de seguridad y representan los límites de tensión en las barras de carga y los límites de flujo de potencia en las ramas. $V_{L i}$ representa la magnitud de la tensión en la barra de carga $i$, mientras que $S_{l i}$ representa el flujo de potencia aparente en la rama i y $N_{K}$ representa el número de ramas del sistema.

$$
V_{g i}^{\min } \leq V_{g i} \leq V_{g i}^{\max }, \quad i=1, \ldots, N_{G}
$$




$$
\begin{array}{ll}
Q_{g i}^{\min } \leq Q_{g i} \leq Q_{g i}^{\max }, & i=1, \ldots, N_{G} \\
T_{i}^{\text {min }} \leq T_{i} \leq T_{i}^{\max } & i=1, \ldots, N_{T} \\
Q_{c i}^{\text {min }} \leq Q_{c i} \leq Q_{c i}^{\max } & i=1, \ldots, N_{c} \\
Q_{r i}^{\min } \leq Q_{r i} \leq Q_{r i}^{\max } & i=1, \ldots, N_{R} \\
V_{L i}^{\min } \leq V_{L i} \leq V_{L i}^{\max }, & i=1, \ldots, N_{G} \\
S_{l i} \leq S_{l i}^{\max }, & i=1, \ldots, N_{K}
\end{array}
$$

\section{MÉTODO DE SOLUCIÓN}

La función objetivo dada por la ecuación (1) y las restricciones (2) y (3) son de tipo no lineal. Adicionalmente, existen variables de tipo entero relacionadas con las inyecciones de reactivos y la posición de los tomas de derivación de los transformadores. En consecuencia, el modelo descrito anteriormente es no lineal, no convexo y multimodal (presenta múltiples soluciones locales). Este tipo de problemas se puede abordar con mayor efectividad mediante técnicas metaheurísticas como ilustrado en (Gómez et al., 2016) y (López-Lezama et al., 2015). En particular, la técnica explorada en este artículo es la optimización por enjambre de partículas (PSO). Esta técnica fue propuesta inicialmente en (Kennedy y Eberhart, 1995). El PSO incorpora aspectos relacionados con el comportamiento social de conjuntos de individuos (bancos de peces, bandadas de aves, enjambres de abejas, etc). Los individuos (que representan candidatos de solución) se mueven en un espacio $n$ dimensional que representa el conjunto de soluciones del problema de optimización. Cada individuo tiene una posición $x_{i}(t)$, y una velocidad $v_{i}(t)$. Además, cada individuo conoce la mejor posición que ha visitado $\left(P_{\text {Best }}\right)$ y la mejor posición del líder $\left(G_{\text {Best }}\right)$. El valor de $P_{\text {Best }}$ trabaja como una memoria autobiográfica (cada individuo recuerda los mejores lugares que ha explorado); por otro lado, $G_{B e s t}$ representa la tendencia de los grupos a visitar lugares donde obtuvieron buenos resultados en el pasado. La selección del líder se realiza en cada iteración. El líder es la partícula que presenta mejor función de adaptación en cada iteración. La función de vuelo y posición de cada partícula en la iteración $t+1$ están dadas por las ecuaciones (11) y (12).

$$
\begin{aligned}
& v_{i}(t+1)=w(t) \cdot v_{i}(t)+c_{1} r_{1}\left(P_{\text {Best }}-x_{i}(t)\right)+c_{2} r_{2}\left(G_{\text {Best }}-x_{i}(t)\right) \\
& x_{i}(t+1)=x_{i}(t)+v_{i}(t+1)
\end{aligned}
$$

En este caso $w(t)$ representa la constante de inercia en la iteración $t$. Dicha constante representa el grado de influencia de la velocidad actual sobre la velocidad futura. $c_{1}$ y $c_{2}$ son constantes de aceleración que representan la influencia de la mejor posición de la partícula y la mejor posición del líder en la nueva velocidad del individuo, respectivamente. $r_{1}$ y $r_{2}$ son números aleatorios en el rango $[0,1]$. El valor de la constante de inercia está dada por la ecuación (13) donde $w_{\max }$ y $w_{\min }$ son los valores máximo y mínimo de $w$; finalmente, $t_{\text {max }}$ es el número máximo de iteraciones.

\begin{tabular}{|c|c|c|c|c|c|c|c|c|c|c|c|c|c|c|c|}
\hline \multicolumn{5}{|c|}{$\begin{array}{l}\text { Tensiones en } \\
\text { Generadores }\end{array}$} & \multicolumn{3}{|c|}{$\begin{array}{l}\text { Bancos de } \\
\text { capacitores }\end{array}$} & \multicolumn{4}{|c|}{$\begin{array}{l}\text { Bancos de } \\
\text { reactores }\end{array}$} & \multicolumn{4}{|c|}{$\begin{array}{l}\text { Tomas en derivación } \\
\text { de transformadores }\end{array}$} \\
\hline$V_{g 1}$ & $V_{g 2}$ & ... & $V_{N G}$ & $Q_{c 1}$ & $Q_{c 2}$ & $\cdots$ & $Q_{N C}$ & $Q r 1$ & Qr2 & $\cdots$ & $Q_{N R}$ & $T_{1}$ & $T_{2}$ & $\cdots$ & $T_{N T}$ \\
\hline
\end{tabular}

$$
w(t)=\left[w_{\max }-\frac{w_{\max }-w_{\min }}{t_{\max }} t\right]
$$

En el ORPD cada candidato de solución se representa mediante un vector de variables de decisión como se ilustra en la Fig. 2. En este caso la primera sección representa las tensiones en las barras de generación que se codifican en rangos discretos desde 0,95 hasta 1,1 en por unidad. Los bancos de capacitores y reactores se codifican en un rango discreto desde 0 hasta 50 MVAr en pasos de 5MVAr. Finamente, las tomas de derivación de los transformadores se codifican según los límites dados por la ecuación (6).

Fig.2: Representación de un candidato de solución 
El diagrama de flujo del PSO se ilustra en la Fig. 3. Se parte de una población inicial generada de forma aleatoria (dentro de los límites de las variables de control). Posteriormente, se calcula la función de adaptación de cada individuo. La función de adaptación consiste en una multiplicación de sub-funciones, cuyo valor máximo es la unidad, como se propone en (Villa et al., 2016). Los límites en las otras variables son controlados directamente en la codificación del algoritmo. Para calcular la función de adaptación se calcula un flujo de carga usando el software Matpower (Zimmerman et al., 2011). Este software recibe los parámetros de entrada de cada individuo correspondientes a la inyección de potencia reactiva de los bancos de capacitores y reactores, la posición de la toma de derivación de los transformadores y las tensiones en las barras de generación. Con esta información se resuelve un flujo de carga que permite calcular las pérdidas del sistema. El procedimiento se hace para cada candidato de solución. Una vez se tienen las pérdidas de potencia asociadas a cada individuo se procede a calcular el $P_{\text {Best }}$ y $G_{\text {Best }}$ y se actualizan los individuos utilizando las ecuaciones (11) a (13). El procedimiento se repite hasta completar un número dado de iteraciones.

Existen algunas variantes del PSO como el Turbulent (TPSO) y el Turbulent Crazy (TCPSO). En ambos casos se redefine la forma en que se actualizan las velocidades de las partículas. En la ecuación (11), si los números aleatorios $r_{1}$ y $r_{2}$ se generan de forma independiente, puede ocurrir que ambos sean muy pequeños o muy grandes. Si son muy grandes la información personal y social de las partículas será supervalorada, posiblemente alejándolas de regiones con buenas soluciones. Por otro lado, si $r_{1}$ y $r_{2}$ son muy pequeños se reduce la velocidad de convergencia. Para evitar este inconveniente se puede utilizar un solo número aleatorio $r_{1}$ y definir $r_{2}$ como 1- $r_{1}$, dando origen a la variante conocida como TPSO. En este caso, la función de vuelo está dada según la ecuación (14).

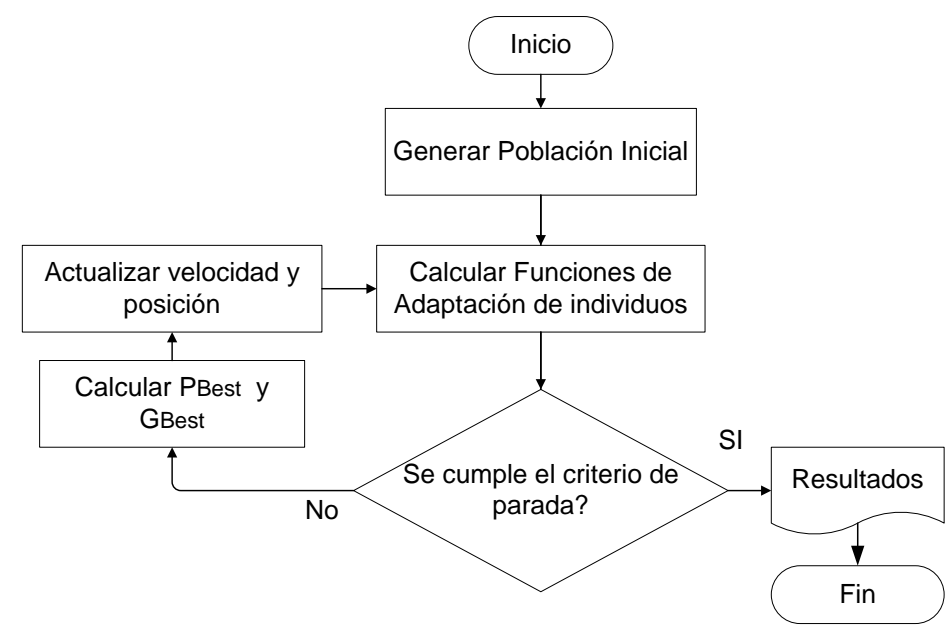

Fig.3: Diagrama de flujo del PSO

$$
v_{i}(t+1)=w(t) \cdot v_{i}(t)+c_{1} r_{1}\left(P_{\text {Best }}-x_{i}(t)\right)+c_{2}\left(1-r_{1}\right)\left(G_{\text {Best }}-x_{i}(t)\right)
$$

La actualización de la velocidad de las partículas también puede hacerse añadiendo un factor que incluya un posible cambio en la dirección de la velocidad como se indica en la ecuación (15) dando origen al TCPSO.

$$
\begin{array}{r}
v_{i}(t+1)=r_{2} \operatorname{sign}\left(r_{3}\right) \cdot v_{i}(t)+\left(1-r_{2}\right) c_{1} r_{1}\left(P_{\text {Best }}-x_{i}(t)\right) \\
+c_{2}\left(1-r_{2}\right)\left(1-r_{1}\right)\left(G_{\text {Best }}-x_{i}(t)\right)
\end{array}
$$

En este caso se define una nueva variable aleatoria $r_{3}$ que toma valores entre cero y uno. La función $\operatorname{sign}\left(r_{3}\right)$ toma el valor 1 (continuar en la misma dirección) solo si $r_{3} \geq 0,05$ y toma el valor - 1 (cambiar de dirección) en caso contrario. También se define un límite mínimo de velocidad dinámico expresado por $v_{c}$ que puede tomar tres valores $\left(v_{c 1}, v_{c 2}\right.$ y $\left.v_{c 3}\right)$ a medida que avanzan las iteraciones. Si en determinada iteración la magnitud de la velocidad es menor que este límite, la actualización de la velocidad se debe hacer de acuerdo con la ecuación (16). La forma en que cambia el límite dinámico se expresa en la ecuación (17) donde $r_{4}$ es una variable aleatoria que toma valores en el rango $[-1,1], v_{\max }$ es la velocidad máxima permitida y $\rho$ es un factor de escalamiento que controla la oscilación de la velocidad. Valores pequeños de $\rho$ hacen que la trayectoria de las partículas tienda a oscilar. Por esta razón, el valor de $\rho$ se incrementa $\left(\rho_{3}>\rho_{2}>\rho_{1}\right)$ a medida que aumentan las iteraciones. Los límites dinámicos de velocidad y el factor de escalamiento permiten al TCPSO un compromiso apropiado entre diversificación e intensificación en la exploración del espacio de búsqueda. 


$$
\begin{aligned}
& v_{i}(t+1)=\frac{r_{4} v_{\max }}{\rho} \quad \text { si }\left|v_{i}(t+1)\right| \leq v_{c} \\
& \left\{\begin{array}{c}
v_{c}=v_{c 1}, \quad \rho=\rho_{1} \quad \text { si } t \leq t_{\max } / 3 \\
v_{c}=v_{c 2}<v_{c 1}, \rho=\rho_{2}>\rho_{1} \quad \text { si } \quad t_{\max } / 3<t \leq 2 t_{\max } / 3 \\
v_{c}=v_{c 3}<v_{c 2}, \rho=\rho_{3}>\rho_{2} \quad \text { si } 2 t_{\max } / 3<t \leq t_{\max }
\end{array}\right.
\end{aligned}
$$

La función objetivo del ORPD es la minimización de pérdidas dada por la ecuación (1). El control de los límites en las variables de decisión (tomas de derivación de transformadores, potencia inyectada por capacitores y límites de tensión en barras de generación) se lleva a cabo directamente en la codificación del algoritmo. Para controlar los límites de tensión en las barras de carga y los límites de flujo en las líneas (ecuaciones (9) y (10)) se utilizó la función de adaptación dada por la ecuación (18). En este caso $f_{V N}(i), f_{C R}(j)$ y $f\left(P_{\text {loss }}\right)$ representan las tensiones en el nodo $i$, el flujo de potencia en la rama $j$ y las pérdidas del sistema, respectivamente. Observe que esta función de adaptación permite establecer un porcentaje máximo aceptable de pérdidas y tiene un límite máximo igual a la unidad cuando todas las variables están dentro de sus límites operativos. Lo anterior permite determinar fácilmente la factibilidad y optimalidad de la solución. Se puede afirmar que una solución es óptima, es decir, cumple con un objetivo previamente establecido, cuando $f(x)=1$. Dado que la función de adaptación es una productoria, esto garantiza que todas las restricciones se cumplen. Lo anterior representa una ventaja desde el punto de vista de optimización, pues se conoce previamente el óptimo global y este valor se puede utilizar como criterio de parada en el proceso de optimización, lo cual reduce el número de iteraciones y tiempo computacional. En la Fig. 4 se ilustra la forma de las sub-funciones que componen la función de adaptación. Una descripción más detallada de la aplicación de esta función de adaptación se puede consultar en (Villa et al., 2016).

$$
f(x)=\prod_{i=1}^{N n} f_{V N}(i) \cdot \prod_{j=1}^{N r} f_{C R}(j) \cdot\left[f\left(P_{\text {loss }}\right)\right]
$$

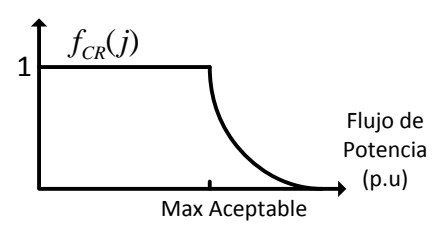

a)

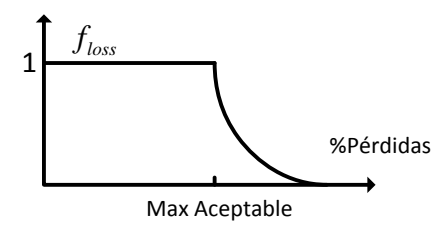

c)

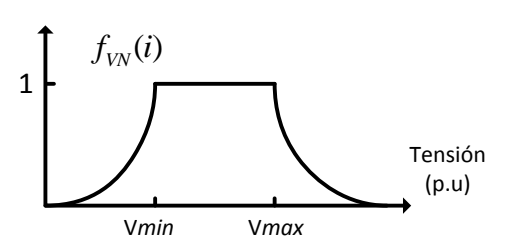

b)

Fig. 4: Componentes de la función de adaptación: a) límites en flujos, b) límites en magnitud de tensión y c) valor máximo de pérdidas aceptable

\section{RESULTADOS}

Para probar la efectividad de la metodología propuesta se utilizaron los sistemas de prueba IEEE de 57 y 118 barras, para los cuales se buscará la gestión óptima de reactivos que minimicen las pérdidas de potencia activa del sistema. Los datos de ambos sistemas se encuentran disponibles en Matpower (Zimmerman et al., 2011). En la Tabla 1 se presenta la información más relevante de estos sistemas. En este caso las abreviaciones Gen., Trans., Cond. y React. significan generadores, transformadores, condensadores y reactores, respectivamente. Se puede observar que las pérdidas iniciales (caso sin optimizar) de los sistemas de 57 y 118 barras es de 27,86MW y 132,45MW, respectivamente. Para aplicar el PSO y sus variantes (TPSO y TCPSO) se calibraron sus parámetros (número de partículas, número de iteraciones, etc) mediante ensayo y error. Para esto se ejecutó varias veces el algoritmo partiendo de valores recomendados en la literatura técnica (Kennedy y Eberhart, 1995). La población inicial se generó de forma aleatoria dentro de los límites de las variables de control. Para todas las pruebas se utilizó una población de 120 partículas y se consideraron 200 iteraciones $\left(t_{\max }\right)$. Los parámetros calibrados fueron: $c_{1}=2,1, c_{2}=2, v_{c 1}=60, v_{c 2}=10, v_{c 3}=1, \rho_{1}=0,1$, $\rho_{2}=0,4, \rho_{3}=0,5, w_{\max }=1, w_{\min }=0,2$. 
Tabla 1: Datos de los sistemas de prueba IEEE

\begin{tabular}{|l|c|c|c|c|c|c|c|}
\hline Sistema & Barras de carga & Gen. & Trans. & Cond. & React. & Líneas & Pérdidas iniciales (MW) \\
\hline IEEE 57 & 50 & 7 & 17 & 3 & 0 & 80 & 27,8637 \\
\hline IEEE 118 & 64 & 54 & 9 & 12 & 2 & 177 & 132,860 \\
\hline
\end{tabular}

Los mejores resultados obtenidos con el PSO y sus variantes se presentan en la Tabla 3. Para ambos sistemas los resultados son comparados con los reportados en (Varadarajan y Swarup, 2008) con las técnicas DE (Differential Evolution) e IPM (Interior Point Method). Para el sistema de 57 barras se comparan también los resultados con los reportados en (Ghasemi et al., 2015) con las técnicas JGGA (Jumping Gen Genetic Algorithm), VEPSO (Vector Evaluated PSO), ITDEA (Interactive Territory Defining Evolutionay Algorithm) y NEKA (Neighborhood knowledge-based evolutionary algorithm).

De acuerdo a los resultados reportados en la Tabla 2 se puede observar que con la versión convencional del PSO, es posible reducir las pérdidas de potencia con respecto a su valor sin optimizar (ver última columna de la Tabla 1). Sin embargo, las mejoras en las pérdidas son marginales y los otros métodos resultan ser más eficaces, pues obtienen soluciones de mejor calidad que el PSO convencional. Por otro lado, al introducir las variantes TPSO y TCPSO es posible obtener mejores resultados que los reportados en (Varadarajan y Swarup, 2008) y (Ghasemi et al., 2015). Esto se debe a que ambas versiones modificadas del PSO introducen perturbaciones dentro del método que obligan a las partículas a hacer una mejor exploración del espacio de búsqueda. Adicionalmente, la definición de la función de adaptación y la estrategia de codificación también influyen en el buen desempeño del algoritmo. En la Fig. 5 se ilustra la convergencia del TCPSO para tres corridas independientes con el sistema IEEE de 118 barras. Se puede observar que la convergencia es estable y el algoritmo alcanza soluciones de alta calidad desde las primeras iteraciones. En las Fig. 6 y Fig. 7 se ilustran los perfiles de tensión de los sistemas IEEE de 57 y 118 barras, respectivamente para la mejor solución obtenida con el TCPSO. Se puede observar que las tensiones están dentro de los rangos permitidos (0,95 a 1,1 p.u). En particular, para el sistema de 118 barras gran parte de las tensiones en las barras de generación se encuentran en su límite superior de 1,1 p.u.

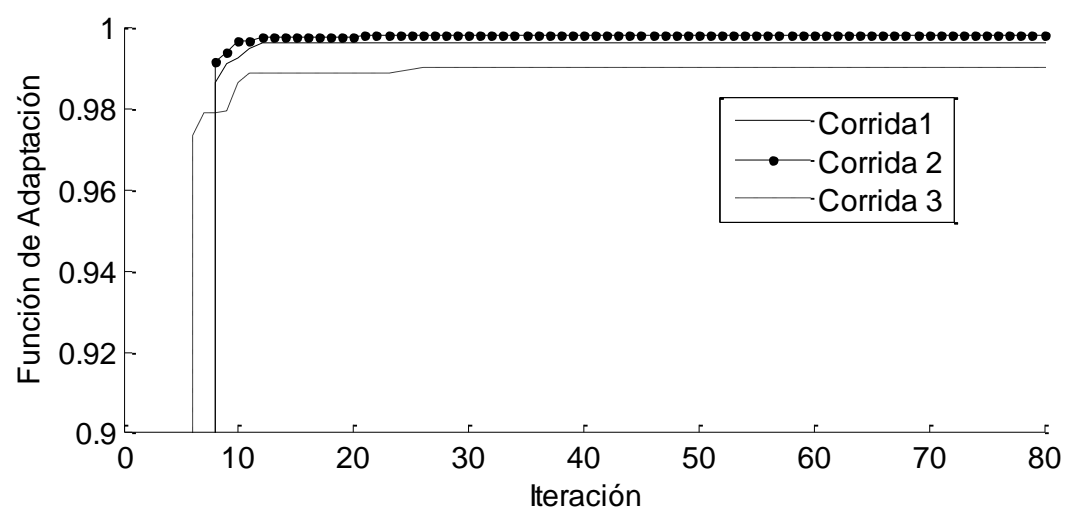

Fig. 5: Convergencia del algoritmo TCPSO para tres corridas independientes

Tabla 2: Soluciones del ORPD con diferentes métodos de optimización (mejores resultados)

\begin{tabular}{|c|c|c|}
\hline Técnica & Pérdidas IEEE 57 barras (MW) & Pérdidas IEEE 118 barras (MW) \\
\hline DE & 25,0475 & 129,579 \\
\hline IPM & 26,6010 & 132,110 \\
\hline JGGA & 25,5318 & - \\
\hline VEPSO & 25,4532 & - \\
\hline ITDEA & 26,7984 & - \\
\hline NEKA & 26,1595 & - \\
\hline PSO & 26,9819 & 130,202 \\
\hline TPSO & 24,2134 & 117,394 \\
\hline TCPSO & 23,9828 & 113,485 \\
\hline
\end{tabular}




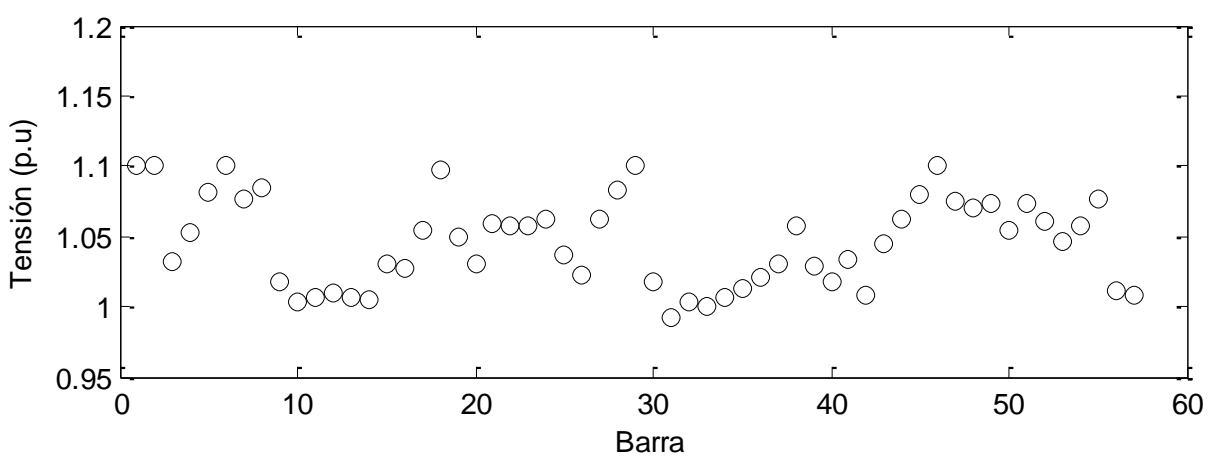

Fig. 6: Perfil de tensiones sistema IEEE 57 barras (mejor solución)

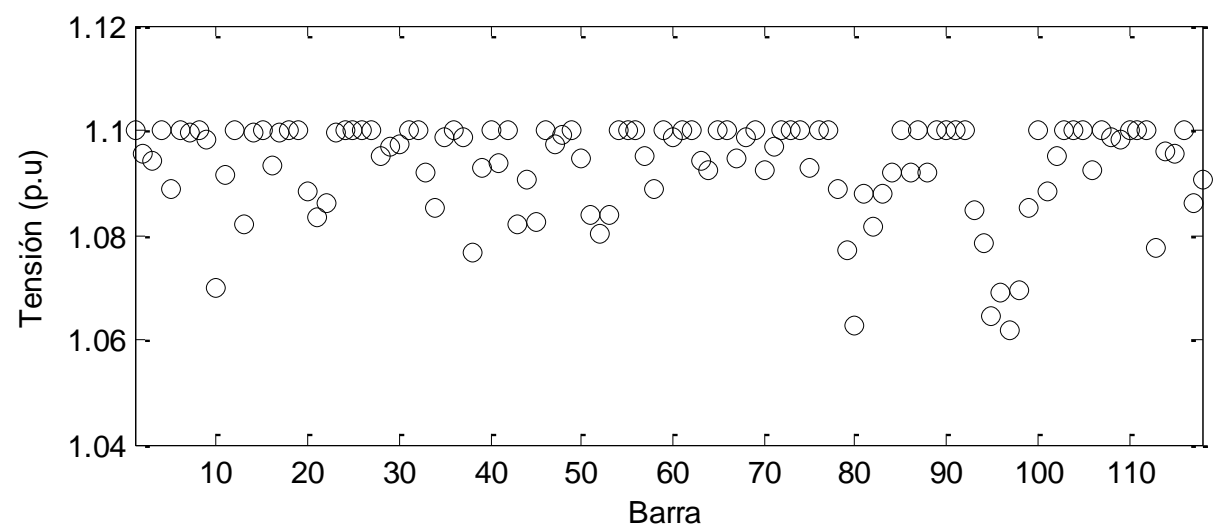

Fig. 7: Perfil de tensiones sistema IEEE 118 barras (mejor solución)

\section{CONCLUSIONES}

En este artículo se presentó un modelo de ORPD solucionado mediante la técnica de optimización de enjambre de partículas y dos de sus variantes. EI ORPD es un problema que presenta no linealidad, no convexidad y múltiples óptimos locales. La técnica propuesta de PSO se comparó con sus variantes TPSO y TCPSO y con otras técnicas reportadas en la literatura especializada: DE, IPM, JGGA, VEPSO, ITDEA y NEKA. Los resultados en sistemas de prueba IEEE de 57 y 118 evidenciaron la aplicabilidad y efectividad de las técnicas bajo estudio.

La comparación de las técnicas de optimización implementadas con las reportadas en la literatura técnica permite concluir que el PSO, en su versión convencional, a pesar de ser competitivo, no es tan efectivo como otras técnicas previamente reportadas. Sin embargo, las versiones TPSO y TCPSO resultan ser mucho más competitivas que el PSO convencional y permiten obtener soluciones de mejor calidad que los reportados con las técnicas DE, IPM, JGGA, VEPSO, ITDEA y NEKA. Esto se debe a que en las variantes TPSO y TCPSO las partículas son sometidas a perturbaciones y también se les permite cambiar de dirección. Esto hace que la exploración del espacio de búsqueda sea más efectiva y le permite al algoritmo escapar de óptimos locales. En un trabajo futuro se puede explorar el desempeño de otras técnicas de solución y considerar otros objetivos en la optimización como el mejoramiento de un índice de estabilidad.

\section{AGRADECIMIENTOS}

Los autores agradecen a la Universidad de Antioquia (UdeA) por el apoyo del proyecto "Sostenibilidad 20162017" para el desarrollo de este trabajo.

\section{REFERENCIAS}

Binod, S.; V. Mukherjee y S.P. Ghoshal. Solution of reactive power dispatch of power systems by an opposition-based gravitational search algorithm, Electrical Power and Energy Systems, 55(1), 29-40 (2014)

Ghasemi, M.; Ghavidel, S.; Ghanbarian, M.M.; Gitizadeh, M. Multi-objective optimal electric power planning in the power system using Gaussian bare-bones imperialist competitive algorithm, Infor. Sciences, 294, 286-304 (2015) 
Gómez, R.A; Giraldo, O.; Campo, E.A. Conformación de Lotes Mínimo Tiempo en la Operación de Acomodo Considerando k Equipos Homogéneos usando Metaheurísticos, Información Tecnológica, 27(6), 53-62 (2016)

Granada M.; López-Lezama, J.M. y Romero, R. An efficient constraint handling methodology for multi-objective evolutionary algorithms, Revista Facultad de Ingeniería, U. de Antioquia, 49(1), 141-150 (2009)

Gutiérrez, D.; López-Lezama, J.M.; Villa, W. Metaheuristic techniques applied to the optimal reactive power dispatch: a review, IEEE Latin America Transactions, 14 (1), 2253-2263 (2016)

Hong, Y.Y.; Lin, F.J.; Lin, Y.C.; Hsu, F.Y. Chaotic PSO-based Var control considering renewables using fast probabilistic power flow, IEEE Transactions on Power Delivery, 29(4), 1666-1674 (2014)

Kanna, B.; Singh, S.N. Towards reactive power dispatch within a wind farm using hybrid PSO, International Journal of Electrical Power and Energy Systems, 69, 232-240 (2015)

Kennedy, J.; Eberhart, R. Particle swarm optimization, Proceedings of the IEEE International Conference on Neural Networks, 4, 1942-1948 (1995)

Khazali, A.; Kalantar, M. Optimal reactive power dispatch based on harmony search algorithm, International Journal of Electrical Power and Energy Systems, 33(3), 684-692 (2011)

Liang, R.H.; Wang, Y.S. Fuzzy-based reactive power and voltage control in a distribution system, IEEE Transactions on Power Delivery, 18(1), 610-618 (2003)

López-Lezama, J.M.; Buitrago L.F. y Villada, F. Ubicación dimensionamiento y precio de contrato óptimo de generación distribuida en sistemas de distribución, Revista Información Tecnológica, 26(6), 109-120 (2015)

Martínez-Rojas, M.; Sumper, A.; Gomis-Bellmunt, O.; Sudria-Andreu, A. Reactive power dispatch in wind farms using particle swarm optimization technique and feasible solutions search, Applied Energy, 88(12), 4678-4686 (2011)

Srivastava, L.; Singh, H. Hybrid multi-swarm particle swarm optimization based multi-objective reactive power dispatch, IET Generation, Transmission and Distribution, 9 (8), 727-739 (2015)

Varadarajan, M.; Swarup, K.S. Differential evolutionary algorithm for optimal reactive power dispatch, Electric Power Systems Research, 30, 435-441 (2008)

Villa, W.M.; López-Lezama, J.M.; Velilla, E. Flujo óptimo reactivo para minimización de pérdidas: una nueva propuesta de función de adaptación y manejo de restricciones, Información Tecnológica, 27(2), 131-140 (2016)

Vlachogiannis, J.G.; Kwang, Y. A comparative study on particle swarm optimization for optimal steady state performance of power systems, IEEE Transactions on Power Systems, 21 (4), 1718-1728 (2006)

Vlachogiannis, J.G.; Lee K.Y. Quantum-inspired evolutionary algorithm for real and reactive power dispatch, IEEE Transactions on Power Systems, 23 (4), 1627-1636 (2008)

Xuexia Z., C.; Weirong y P.N. Suganthan, Optimal multi-objective reactive power dispatch considering static voltage stability based on dynamic multi-group self-adaptive differential evolution algorithm, $2^{\text {th }}$ Conference on Intelligent System Design and Engineering Application (ISDEA), 1448-1456 (2012)

Zimmerman, R.; Murillo, C.; Thomas, R. Steady-State Operations, Planning, and Analysis Tools for Power Systems Research and Education, IEEE Transactions on Power Systems, 26 (1), 12-19 (2011) 
